\section{Mandibular cortical bone height in bruxism patients based on gonion and antegonial index}

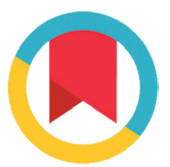

CrossMark

\author{
Fidela Dwirahma, ${ }^{1 *}$ Setyawan Bonifacius, ${ }^{1}$ Lusi Epsilawati ${ }^{2}$
}

\title{
Abstract
}

Objective: To determine differences in the height of the mandibular cortical bone in patients with bruxism and those who were not.

Material and Methods: This study used an analytic method with a cross-sectional approach. Samples taken were divided into two groups, each with 30 digital panoramic radiograph samples of people with bruxism and those not with bruxism. The study was conducted by measuring the height of the mandibular cortical bone on digital panoramic radiograph based Gonion Index (GI) and Antegonial Index (Al) using Image J application.
Results: Bruxism patients aged 21-30 years were 27 people and aged 31-40 years were 3 people, with 12 male samples, and 18 female samples. Samples of non-bruxism patients aged 21-30 years were 28 people and aged 31-40 years were 2 people, with 15 male samples and 15 female samples.

Conclusion: There was no difference in the height of the mandibular cortical bone in patients with bruxism and non-bruxism patients based on $\mathrm{Gl}$ and $\mathrm{Al}$.
${ }^{1}$ Department of Prosthodontics, Faculty of Dentistry, Padjadjaran University, Bandung, Indonesia ${ }^{2}$ Department of Oral and Maxillofacial Radiology, Faculty of Dentistry, Padjadjaran University, Bandung, Indonesia

${ }^{*}$ Correspondence to:

Fidela Dwirahma, Department of Prosthodontics, Faculty of Dentistry, Padjadjaran University, Bandung, Indonesia

fidela16001@mail.unpad.ac.id

Received: 30 March 2020

Revised: 17 July 2020

Accepted: 17 March 2021

Keyword: Antegonial index, Bruxism, Gonion index, Mandibular cortical bone, Remodeling

Cite this Article: Dwirahma F, Bonifacius S, Epsilawati L. 2021. Mandibular cortical bone height in bruxism patients based on gonion and antegonial index. Journal of Dentomaxillofacial Science 6(3): 156-160. D0l: 10.15562/jdmfs.v6i3.1046

\section{Introduction}

Bruxism is listed as the most common parafunctional habit of the dentofacial system. ${ }^{1}$ In the United States, it is estimated that as many as 45 million people have signs and symptoms of bruxism during sleep and $20 \%$ of the population experiences bruxism upon awakening. ${ }^{2} 8 \%$ of adult population and $3 \%$ in the elderly. According to the existing literature, two groups of proposed etiological factors can bedistinguished: peripheral (morphological The prevalence of bruxism will decrease with age. ${ }^{3}$

Frank Lobbezoo et $\mathrm{al}^{4}$ described bruxism as a repetitive jaw muscle activity characterized by clenching or grinding of the teeth and/or by bracing or thrusting of the mandible. Grinding and clenching movements of teeth when bruxism can cause a large and continuous force/pressure exerted on bone structure and tooth-supporting tissues that leads to trauma occlusion. ${ }^{5}$ Occlusal forces can cause changes in the alveolar bone by resorption and bone apposition, altering the height, number, and thickness of the trabeculae. ${ }^{6}$

The diagnosis of bruxism can be made through examinations which include subjective, objective, radiological examinations and additional examinations. ${ }^{7}$ Panoramic radiography is one type of extraoral radiography that can be used because it has wide applications as complementary clinical radiology in dentistry. ${ }^{8}$ The panoramic radio morphometric index can detect the structural changes in the maxilla and mandible. ${ }^{9}$ A number of mandibular cortical indices, including Antegonial Index (AI) and gonial index (GI) have been developed to assess and measure the quality of mandibular bone mass and to observe signs of bone resorption. ${ }^{10}$ In our study, we have chosen AI and GI to measures the thickness of the cortical mandible.

\section{Material and Methods}

This study uses analytical research with crosssectional study design or prevalence research. The population of this study was all panoramic radiography of Dental Hospital Padjadjaran University patients who were bruxism and non-bruxism in terms of medical records. The inclusion criteria for sampling in this study include: digital panoramic radiographs of patients with bruxism and nonbruxism patients who have complete teeth up to the second molar teeth, amounting to 32 teeth, digital panoramic radiography of bruxism patients and non-bruxism patients aged 20-40 years old. Exclusion criteria in the study include: there is a picture of radiopaque and/or radiolucent, due to fractures or pathological abnormalities, in the measurement area, there are superimposed in the measurement area, patients with supernumerary teeth, mandibular canal not clear.

The method of sampling is a total sampling. The samples used were 30 samples of panoramic radiographs of bruxism patients and 30 samples of panoramic radiographs of non-bruxism patients. Samples were obtained from the previous study at the Radiology Installation of Dental Hospital 
Padjadjaran University. The tools and materials needed in this study are computers, panoramic radiography archives, ImageJ software applications, and Megastat applications. The time of the study was conducted for 2 months starting from NovemberDecember 2019, at the Prosthodontic Installation and Radiology Installation of Dental Hospital Padjadjaran University, Bandung. Bone height in this study was measured using the GI and AI. AI is a measurement of cortical width in the anterior region to the gonion at the point identified by extending the most suitable line at the anterior border of the ascending ramus to the lower border of the mandible. GI is measured by drawing a vertical tangent to the posterior border of the ramus, the angle made by this line with a tangent to the lower border of the mandible in the mental foramen halved. at the intersection of these bisector lines with the mandibular angle, the thickness of the angular cortex is measured. Statistical analysis in this study uses the normality test with chi-square, to see whether the sample data distribution is normal or not. The homogeneity test was carried out using the $\mathrm{F}$ test variance (Levene's Test). Independent sample T-Test was conducted to see differences in the height of the mandibular cortical bone in patients with bruxism and non-bruxism.

\section{Results}

The 60 radiograph samples obtained are distinguished based on their characteristics. Table 1 distinguishes samples of bruxism and non-bruxism based on age and sex. Bruxism patients ag ed 21-30

Table 1 Characteristics of research subjects

\begin{tabular}{lccccccc}
\hline \multirow{2}{*}{ Variable } & \multicolumn{3}{c}{ Age } & & \multicolumn{3}{c}{ Gender } \\
\cline { 2 - 3 } \cline { 6 - 7 } & $\mathbf{2 1 - 3 0}$ & $\mathbf{3 1 - 4 0}$ & & Male & Female & Total \\
\hline Bruxism Patients & 27 & 3 & & 12 & 18 & 60 \\
Non-Bruxism & 28 & 2 & & 15 & 15 & 60 \\
Patients & & & & & & \\
\hline
\end{tabular}

Table 2 Average measurement of height of mandibular cortical bone in bruxism patients and non-bruxism patients based on gonion and antegonial index

\begin{tabular}{lcccccc}
\hline & \multicolumn{2}{c}{ Gonion Index } & & & \multicolumn{3}{c}{ Antegonial Index } & \\
\cline { 2 - 3 } Regio & Bruxism & $\begin{array}{c}\text { Non- } \\
\text { Bruxism }\end{array}$ & Difference & Bruxism & $\begin{array}{c}\text { Non- } \\
\text { Bruxism }\end{array}$ & Difference \\
\hline Right & 2,100 & 2,166 & 0,066 & 4,373 & 4,147 & 0,226 \\
Left & 2,058 & 2,398 & 0,340 & 4,257 & 3,959 & 0,298 \\
Average & 2,079 & 2,282 & 0,203 & 4,315 & 4,053 & 0,262 \\
\hline
\end{tabular}

years were 27 people and aged 31-40 years were 3 people, 12 male samples and 18 female samples. Samples of non-bruxism patients aged 21-30 years were 28 people and aged $31-40$ years were 2 people, with 15 male samples and 15 female samples.

Table 2 shows the average results of measuring the height of the mandibular cortical bone in patients with bruxism and non-bruxism patients based on the Gonion Index (GI) and Antegonial Index (AI). The measurement results using GI, the average height of the mandibular cortical bone in patients with bruxism is smaller than the group of non- bruxism patients with $0.203 \mathrm{~mm}$ difference.

The results of measurements using $\mathrm{AI}$, the average height of the mandibular cortical bone in patients with bruxism is greater than the nonbruxism group with a $0.262 \mathrm{~mm}$ difference.

The normality test results as shown in table 3 show the p-value obtained from each mandibular cortical bone height calculation data based on the Gonion and Antegonial Index is greater than the significant level that has been determined, which is 0.05 . This shows that the data obtained are normally distributed, so then homogeneity tests or variance tests are performed with the F- Test or Levene's Test.

Table 4 shows the significance values of the left and right mandibular cortical bone height based on the Gonion and Antegonial Index greater than 0.05 . The conclusion is based on the homogeneity test above, that the data variance from the mandibular cortical bone height measurement is the same or homogeneous.

Table 5 shows the results of the p-value of $0.3096>\alpha=0.05$ and $0.30001>\alpha=0.05$, according to the hypothesis testing criteria, if $\mathrm{p}$-value $>\alpha$ then Ho is accepted which shows no significant difference between mandibular cortical bone height in patients with bruxism and non-bruxism patients based on the GI in terms of panoramic radiographs.

Table 6 shows the results of the p-value of $0.0916>a=0.05$ and $0.2220>a=0.05$, according to the hypothesis testing criteria, if $\mathrm{p}$-value $>\alpha$ then Ho is accepted which shows no significant difference between mandibular cortical bone height in patients with bruxism and non-bruxism patients based on the $\mathrm{AI}$ in terms of panoramic radiographs.

\section{Discussion}

Changes in mandibular height can be influenced by many factors, including the presence of traumatic occlusion due to bruxism. Some studies show that occlusion trauma can have destructive pathological effects on the periodontium, masticatory muscles, 
Table 3 Normality test

\begin{tabular}{lcc}
\hline & \multicolumn{2}{c}{ Normality Test Results } \\
\cline { 2 - 3 } Variable & p-value & Distribution \\
\hline G-Ka-B & 0.1870 & Normal \\
G-Ki-B & 0.3080 & Normal \\
A-Ka-B & 0.6594 & Normal \\
A-Ki-B & 0.4060 & Normal \\
G-Ka-N & 0.1577 & Normal \\
G-Ki-N & 0.2214 & Normal \\
A-Ka-N & 0.1328 & Normal \\
A-Ki-N & 0.2615 & Normal \\
\hline
\end{tabular}

A value of $p>0.05$ indicates that the data is normally distributed

Table 4 Homogeneity test

\begin{tabular}{lccc}
\hline Variable & F & p-value & Trait \\
\hline G-Ka & 1.19 & 0.32364 & Homogeneous \\
G-Ki & 1.10 & 0.399586 & Homogeneous \\
A-Ka & 1.08 & 0.419644 & Homogeneous \\
A-ki & 1.04 & 0.456796 & Homogeneous \\
\hline
\end{tabular}

Table 5 Results of independent samples analysis t tests on cortical mandibular bones of bruxism patients and non-bruxism patients based on gonion index

\begin{tabular}{lcc}
\hline Place of Measurement & p-value & Conclusion \\
\hline Right Mandible & 0.3096 & The difference is not significant \\
Left Mandible & 0.3001 & The difference is not significant \\
\hline
\end{tabular}

Table 6 Results of independent samples analysis $t$ tests on cortical mandibular bones of bruxism patients and non-bruxism patients based on antegonial index

\begin{tabular}{lcc}
\hline Place of Measurement & p-value & Conclusion \\
\hline Right Mandible & 0.0916 & The difference is not significant \\
Left Mandible & 0.2220 & The difference is not significant \\
\hline
\end{tabular}

tooth structure, TMJ, and alveolar bone. The alveolar bone reaction to the occlusal force depends on the direction, intensity and duration of the force. The occlusal force forms alveolar bone by reshaping bone, in the form of bone resorption and apposition, changing the height, number, and thickness of the trabeculae. ${ }^{11}$ In normal bone remodeling, the balance between bone resorption and bone formation is tightly regulated and maintained by coupling bone formation with bone resorption, which involves a number of coordinated signaling mechanisms to ensure that, in healthy adult bone, there is no major change in bone mass or mechanical strength after each cycle remodeling. An imbalance can occur in certain pathological conditions, such as hormonal changes related to menopause, age-related factors, changes in a mechanical activity such as bruxism, drugs, and secondary diseases. The force due to occlusion trauma, which can be caused by bruxism, is then considered as one of the local factors that accelerates bone resorption. ${ }^{12}$

Shows a higher sample of sufferers of bruxism in women than men, this may be due to psychosocial factors such as state anxiety and trait anxiety, alexithymia, and stress which are factors that cause awake bruxism. A Japanese population study by Nakata et al. ${ }^{12}$ concluded that bruxism was less related to work-related stress in men, but not in women. Data in specialist literature also shows that women suffer more bruxism than men. ${ }^{13}$

Samples taken in this study have criteria of age above 20 years and under 50 years. Table 1 shows that 27 samples of bruxism patients were in the age range of 20-30 years. This is consistent with Khoury et $\mathrm{al}^{3}{ }^{3}$ statement that the prevalence of bruxism decreases with age. The prevalence of Sleep Bruxism (SB) tends to decrease with increasing age with a positive SB report is 30\% at 18-34 years, $25 \%$ at $35-54$ years, and $14 \%$ at $55+$ years.

Shows the average results of the height of the right and left cortical mandibular bones in patients with bruxism and non-bruxism patients based on the Gonion Index (GI) and Antegonial Index (AI) which have mathematical differences in values. Statistically, after analyzing using independent samples t-test, in Tables 5 and 6 the results show that the p-value of all tests carried out are all more than 0.05 which means that Ho is accepted and also means that there is no significant difference between mandibular cortical bone height of bruxism patients and non-bruxism patients both based on GI and AI. According to several studies, bone remodeling is calculated more often in the jaw, especially in the intracortical component. ${ }^{14}$ The level of remodeling in trabecular bone is proportionally more common, usually 5-10 times higher than cortical bone remodeling. The resistance of bone tissue to compressive forces is proportional to the square of its density; modulus of elasticity, as a result, the mechanical resistance of cortical bone can reach 10 times that of trabecular bone. Trabecular bone occupies about 20 times more surface area per unit volume than cortical bone, and therefore, its cells can be more easily and directly affected by forces. These facts show cortical bone has a lower metabolic capacity and remodeling activity compared to alveolar bone. ${ }^{15}$

Murali et al. ${ }^{16}$ mention the existence of exostosis as one of the clinical signs of bruxism. Exostosis is a small, non-pathological hyperplasia area and is localized to the cortical bone and sometimes 
the internal cancellous bone in the alveolar. The etiology is unknown, but some authors consider it to be supporting bone formation in response to trauma due to occlusion and suggest that it occurs to strengthen bone trabeculae to support teeth and functional adaptation. Exostosis is more common in the maxilla than in the mandible with a ratio of 5:1. ${ }^{17}$ This explanation is in accordance with previous theories regarding the strength of the mandibular cortical bone.

The mastication system is designed to eliminate occlusal loads, where periodontal ligaments and adjacent alveolar sheaths are closely correlated in the distribution of masticatory forces. The periodontal ligament functions as a viscoelastic shock absorber because it functions like a hydraulic system that functions to reduce the amount of force and pressure applied to the bone. When "occlusion trauma" occurs, the tooth will respond by increasing its mobility to eliminate stress and tension on the bone surface. ${ }^{18}$

Histologically, teeth affected by occlusal trauma show different zones of tension and pressure in adjacent periodontal tissue. ${ }^{19}$ Comparing occlusal forces distribution to the tooth roots and supporting bones. They found that stress was higher in the cervical area and lower in the apical teeth. Periodontal ligament fibers (PDL) can protect and influence the response of the bone due to the occlusal force, if the value of the occlusal strength exceeds the resistance capacity of the periodontal ligament, then the alveolar bone can be destroyed, but the ability to apply its stress force (strain) is not enough to trigger the mechanical adaptation of cortical bone. The maximum stress that PDL fibers can provide to the bone is not enough to create the stretch gradient that the bone needs to adapt. PDL fiber tensile strength will create a maximum strain (strain) between $133 \mathrm{~m} . \mathrm{s}$ and $210 \mathrm{~m} . \mathrm{s}$. The range of strain needed for bone adaptation should be between 200-1500 m.s for and as high as $2500 \mathrm{~ms}$ for positive and negative normal strain (compressive). The above facts can then be concluded, that the PDL fibers abilities to cause changes in cortical bone seems limited. ${ }^{20}$

The results of a research analysis of 60 radiographic samples of bruxism patients and nonbruxism patients based on the Gonion and Antegonial Index showed an average difference in cortical bone height of only $0.23 \mathrm{~mm}$ and there were no statistically significant differences. This study has several limitations in its implementation namely, the use of secondary data makes the data obtained about the patient limited, such as the intensity of the patient's bruxism and the duration of bruxism has occurred.

\section{Conclusion}

Based on the results of research conducted it can be concluded that there is no statistical difference between the height of the mandibular cortical bone in patients with bruxism compared with nonbruxism patients based on the Gonion and antegonial Index in terms of panoramic radiography.

\section{Acknowledgment}

Thank you for all support Faculty of Dentistry Padjadjaran University.

\section{Conflict of Interest}

The authors report no conflict of interest.

\section{References}

1. Kanathila H, Pangi A, Poojary B. Diagnosis and treatment of bruxism : concepts from past to present. Int J Appl Dent Sci 2018;4: 290- 295.

2. Hartono SWA, Rusminah N, Adenan A. Bruksisma bruxism. J Dentomaxillofac Sci 2011;10: 184.

3. Torres S, Chen C, Leroux B. Mandibular inferior cortical bone thickness on panoramic radiographs in patients using bisphosphonates. Oral Surg Oral Med Oral Pathol Oral Radiol 2015;119: 584- 592.

4. Ahlberg J, Glaros AG, Kato T, et al. Oral rehabilitation bruxism defined and graded: an international consensus. J Oral Rehabil 2012;40: 1- 9.

5. Satheeswarakumar LP, Elenjickal TJ, Kiran S, et al. Assessment of mandibular surface area changes in bruxers versus controls on panoramic radiographic images : a case control study. Open Dent J 2018;12: 753- 761.

6. Ispas A, Craciun A, Kui A, et al. Effects of occlusal trauma on the periodontium, alveolar bone, temporomandibular joint and central nervous system. Hum Vet Med Int J Bioflux Soc 2018;10: 158- 162.

7. Kurnikasari E. Berbagai teknik penanganan bruksisme. J Mater Kedokt Gigi 2013;2: 36- 42.

8. Okșayan R, Asarkaya B, Palta N, et al. Effects of edentulism on mandibular morphology: evaluation of panoramic radiographs. Sci World J 2014;2014.

9. Mamadou Lamin N, Pape Abdou L, Mamadou D, Augustin V, Babacar T. Radiographic evaluation of mandibular bone remodeling by mandibular cortical Index (MCI) in a Senegalese population. J Oral Med Oral Surgery Oral Pathol Oral Radiol 2018;4: 136- 138

10. Bajoria AA, Ml A, Kamath G, et al. Evaluation of Radiomorphometric indices in panoramic radiograph - a screening tool. Open Dent J 2015;9: 303- 310.

11. Feng X, Mcdonald JM. Disorders of bone remodeling. Annu Rev Pathol 2011;6: 121- 145.

12. Nakata A, Takahashi M, Ikeda T, et al. Perceived psychosocial job stress and sleep bruxism among male and female workers. Community Dent Oral Epidemiol 2008;36: 201- 209.

13. Przystańska A, Jasielska A, Ziarko M, et al. Psychosocial predictors of bruxism. Biomed Res Int 2019;2019: 15- 22.

14. Khoury S, Carra MC, Huynh N, et al. Sleep bruxism-tooth grinding prevalence, characteristics and familial aggregation: a large cross-sectional survey and polysomnographic validation. Sleep 2016;39: 2049- 2056.

15. Brandini D, Debortoli C, Felipe-Akabane S, et al. Systematic review of the effects of excessive occlusal mechanical load on the periodontum of rats. Indian J Dent Res. 2018;29: 812-819. 
16 Murali R V, Rangarajan P, Mounissamy A. Bruxism : conceptual discussion and review. J Pharm BioAllied Sci 2015;7: 265- 270.

17 Isha M, Ramaswami E. Bony exostoses : case series and review of literature. Acta Sci Dent Sci 2018;2: 64- 67.

18. Graves CV, Harrel SK, Rossmann JA, et al. The role of occlusion in the dental implant and peri-implant condition: a review. Open Dent J 2016;10: 594- 601.

19. Fan J, Caton JG. Occlusal trauma and excessive occlusal forces: Narrative review, case definitions, and diagnostic considerations. J Clin Periodontol 2018;89: 214- 222.
20. Ben-Yehouda A. Occlusal load transmission to bone via the periodontal attachment apparatus. J Dent Implant 2012;2: 88- 92 .

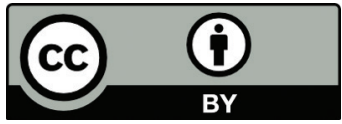

This work is licensed under a Creative Commons Attribution 